\title{
The Study on Heuristic and Algorithmic Processing Creative Training on Product Design Education: Based on the Bags \& Luggage design Course
}

\author{
Ziran Zhang ${ }^{1}$, Ying Yu ${ }^{2}$, Shengxi Fan ${ }^{3}$ and Yujing Tian ${ }^{4}$ \\ ${ }^{1}$ Shanghai University of Engineering Science, School of Art and Design, 201620 Shanghai, P.R. China \\ ${ }^{1}$ Tongji University Shanghai Institute of Design and Innovation, School of Design, 200092 Shanghai, P.R. China \\ ${ }^{1}$ Tongji University, College of Design and Innovation, 200092 Shanghai, P.R. China \\ ${ }^{2}$ Donghua University, Fashion and Design College, 200051 Shanghai, P.R. China \\ ${ }^{3}$ Tongji University, College of Design and Innovation, 200092 Shanghai, P.R. China \\ ${ }^{4}$ Donghua University, Fashion and Design College, 200051 Shanghai, P.R. China
}

\begin{abstract}
In the study, context-creativity of Teresa M. Amabile was used as the foundation to apply it in the bags \& luggage design course. Moreover, the sectional creative training education mode of prior heuristic task and postpositional algorithmic task was proposed. 26 junior students in product design were used as the trial objects. The Consensual Technique for Creativity (CAT) was considered as the scoring standard of creative performance. In the end, the sectional theoretical framework of effective creative training in product design was finally proposed.
\end{abstract}

\section{The relationship between context-creativity theory and product design}

\subsection{Based on context-creativity theory}

Differing from relevant psychological studies that creativeness or creativity is equal to divergent thinking ability test[1], respondents participate in actual activities in the experiment conducted by Teresa M. Amabile, such as barbola, prose creation and storytelling. This is consistent with the final purpose-ending of practice problems in design subject. The achievement is really visible and evaluable[1]. In early studies, Amabile created a test technology to evaluate whether works could own some creativity. And this is called as Consensual Technique for Creativity (CAT)[2](It is a relative science and repeatedly verified subjective evaluation test). This actually has a great similarity with subjective judgment for works. Even if most of design teachers don't verify scoring and evaluation form for works creativity, CAT has more practical value for the quantized creative test in psychological study conducted in other labs(such as TTCT)[3]. Moreover, it is closer to subjective evaluation method for single works in artistic design subject. The research conclusions are more suitable for theoretical foundation in artistic design subject education methods.

\subsection{Algorithmic Processing and Heuristic Processing in Product Design Process}

\footnotetext{
${ }^{1}$ Corresponding author: zhangziran_080@126.com
} 
T.M. Amabile repeatedly proposes an idea that creative performance of external limitations in the algorithmic task and heuristic task is entirely different in Context Creativity. Artistic creation lays particular stress on algorithm in technologies, while creativity focuses on heuristic[2] (The algorithmic and heuristic concepts will be stated in details as follows). Similar to creative process of artistic works, designers control technical knowledge and creative design of products in product design process, so as to create a new product. As a result, training on "technologies" and "creativity" is the teaching target in product design major. The commonly used basic design method and process of professional studies in integrated product design course is shown in the right side of Figure 1[4]. It can be clearly observed from the figure that as training students in product major, creative inspiration(conceptual extraction and creativity in the figure) is included, and practical operation of relevant technologies(product formation and achievement publication) is also contained. After studying every part, it can be clearly shown that creative inspiration also contains knowledge in technical levels, such as market investigation, sketch and analysis of functional structure. Based on the context-creativity theory, these contents should belong to the algorithmic processing mode. The influence factor is totally different from the heuristic processing mode, thus the training method is also diverse.

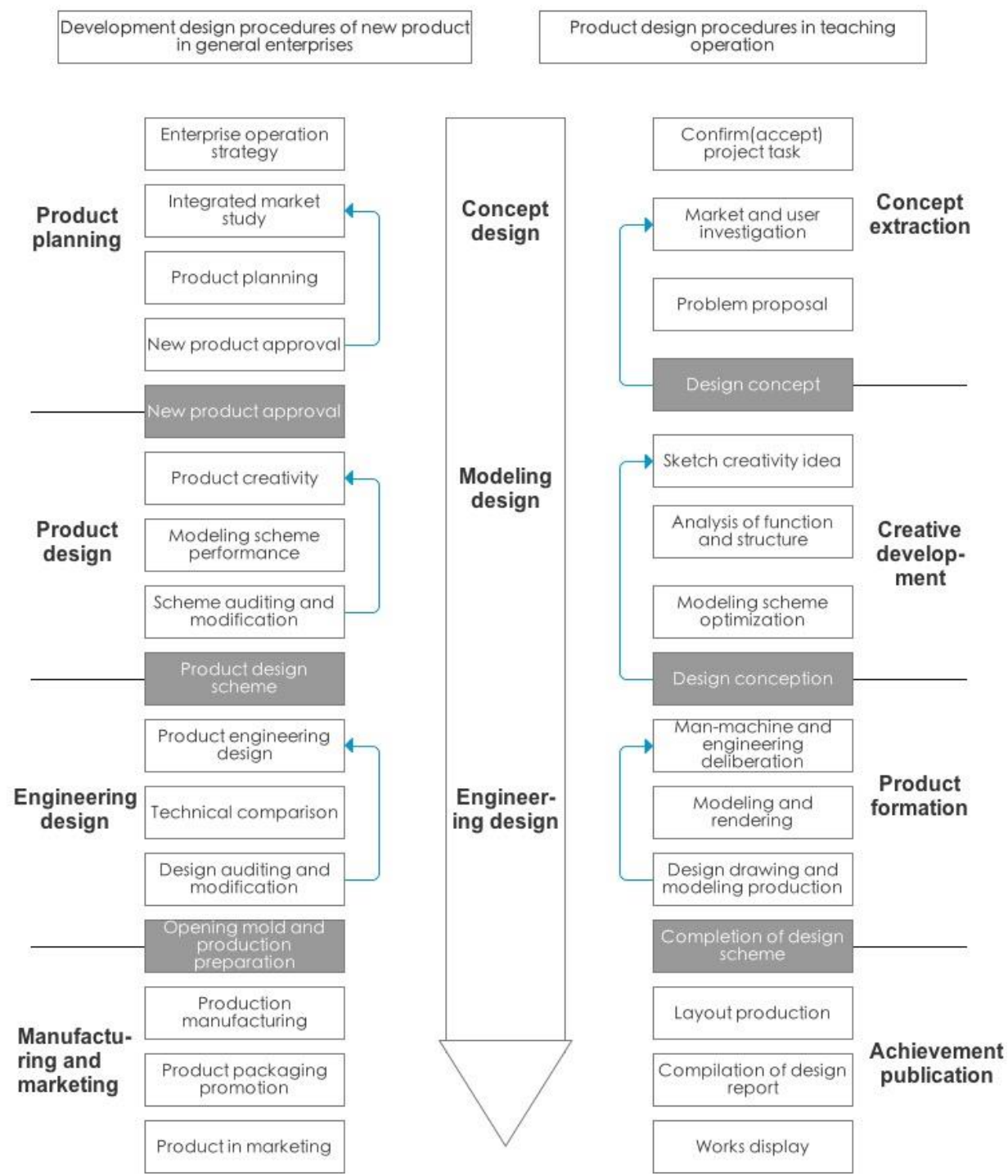


Figure 1. Comparison between Development Design Process of New Products in General Enterprises and Product Design Procedures in Teaching Operation [4]

\subsection{The Research Object of the Bags \& Luggage Design Course}

There is a large dispute in professional attribution of the bags \& luggage design course. Some colleges in Chinese mainland classify it in clothing major. Some of them are divided into the product major, because this is the impression on general classification of products(such as supermarket, market and online shopping platform). Generally speaking, these products are displayed to consumers as garment accessories. From the perspective of processing technology, it is exactly the same with relevant technical knowledge of products in ordinary meanings for luggage structure parts and production technologies, especially for hard cover luggage. As a result, it has no need to doubt whether bags \&luggage course has representativeness in the product design course system. The truth that we design in the study means that heuristic processing and algorithmic processing in product design task implementation are relative key points on how to cultivate creativity. Therefore, the differences of diverse courses in technical knowledge, creative training contents, degree and depth won't affect preliminary conclusion of the empirical study. Relative to furniture, automobiles and household appliances, technical knowledge contents of luggage design are more intuitive and easier to be mastered, so that students are able to reach the required baseline level in a short time( 32 hours in the study)[2]. According to three basic requirements in CAT[5] proposed by Ambile, the third one means that research objects shouldn't have the large individual difference in baseline performances. As a result, the trail should improve research objects to the same baseline level in the shortest time of the course teaching. Thus, it can be observed that relative to other products that have the relatively complicated technologies and materials, the bags \& luggage design course conforms to the required conditions of the trial.

\section{Necessity of Sectional Training}

\subsection{Differences between “Algorithmic” Processing and "Heuristic” Processing}

Amabile found in the pasteup task in 1979 that when the "creative" solution in the task can't figure out steps in task development, the creative performance of respondents will be easier to be affected by external limitation and form the negative correlation. In other words, the more obvious limitation is, the lower creative scores of respondents will be. On the contrary, for respondents of technical scoring, when they are provided with definite information of technical concern and generate evaluation expectations, the technical level scoring will be higher than relevant control group[2], showing that technical level of artistic works will be slightly improved in definite scoring concern and evaluation expectation on respondents. Such a process is called as the "algorithmic" processing. In the "heuristic" processing, task type must be an open method without standard answers or obvious solutions. Secondly, external limitation in task implementation should be prevented or weakened. For example, respondents shouldn't generate evaluate expectation and avoid from passive effects of awards on creative performance.

\subsection{Sectional Training and Definite Teaching Methods}

Educators often insert thinking training and relevant technical knowledge in previous design education according to their experience(shown in Figure 1), but it is not systemic and scientific. Moreover, sectional teaching method points are not definite. Homework setting has no strong purposefulness to how to enhance creative performance. Reward mechanism is also applied at random. Evaluation proposal is also mastered by virtue of personal experience. There is no scientificity and demonstration support. As teachers, we often show the clear marking criterion and rewarding mode to students at the 
beginning of courses and then start the specific step implementation of courses. Specific contents will be based on market investigation and divergent thinking training shown in Figure 1(especially for brainstorming [6]). Then, comments and technical knowledge are inserted to carry out the specific design procedures. The research contents of Amabile show that rewards and external limitations will be helpful to enhancement of technical level. As the creative conception dominated by the heuristic processing, too early or overemphasis reward mechanism will reduce the creativity. By aiming at totally different conclusions, the sectional teaching method was put forward in the study to divide product design teaching process into three steps: (1) prior heuristic task; (2) postpositional algorithmic task; (3) concrete implementation of heuristic task achievements. At the same time, by aiming at two types of task process, people formulate the relevant teaching mode in accordance with the different creative reaction mode performed by external limitation.

\subsection{Universality of the Sectional Teaching Method in the Product Design Course}

The teaching method proposed in the paper was proposed in line with the context-creativity theory by Amabile. In the trial, 26 undergraduates in product design major were selected as observational and interview objects of the empirical study. Due to limitation of sample quantity and implementation in the same school, the preliminary exploration was only formed in the theoretical framework. Later, the more extensive empirical study on different courses of product design in different colleges should be conducted, so as to prove universality of the method.

\section{Trail}

\subsection{Trail subjects and contents}

This trial is a preliminary attempt of the distributed teaching method. By selecting 26 undergraduate students of Grade Three majoring in product design from a famous university of Shanghai, China as the research subjects, and dividing the content of the bag product design course into three stages: the heuristic task stage, the algorithmic task stage and the implementation stage of heuristic task outcomes, it aims to improve the creative performance of students when designing works through the application of Amabile's theory of Creativity in Context.

26 students in the trial were divided into 2 groups and they were sampled according to their student number. Students with odd student number were the control group, and students with even student number were the trial group. Two groups of students participated in the first two stages. Only in the final concrete implementation stage of task outcomes, the content of the trial was different, so two groups of subjects were tested separately. In the final stage, the trial group was asked to further design for the creativity in the heuristic stage and put it into a specific design plan. The subjects in the control group were asked to design an arbitrary concept suitcase or bag and show the design plan. Two different instructions were used to test whether the segmented education was effective in enhancing the creativity of the subjects. The former was the effective segmented education method that we assumed, and the latter was a proposition designed to weaken the influence of the heuristic process in the final product design. Finally, works of the two groups were merged and randomly sorted and handed over to the judges for the creativity of the works. (The judges didn't know the difference between the groups).

\subsection{The heuristic task first}

\subsubsection{Heuristic Task}

The heuristic task is a type of task that is quite different from the algorithmic task. The results of such a task should be sufficiently open to ensure that the response has considerable flexibility and 
innovation. [2] The task must have certain openness, and it needs some exploration to get the solution. Moreover, the idea of getting the right solution is less clear. At the same time, the task should be designed to prevent the task executor from being transformed into an algorithmic task. When $a$ heuristic task is converted to an algorithmic task, subjects perform better under external constraints. [2] Since heuristic tasks are sometimes transformed into algorithmic tasks, how to design propositions for heuristic tasks and how to reduce the generation of such transformations has become the most important focus.

\subsubsection{Heuristic task first}

Baseline level of subjects. Since the selected subjects are third-grade students majoring in product design, they have not studied any design method courses related to the design of bag products before taking this course. Therefore, they can be regarded as subjects at the same baseline level, with a certain way of thinking theory, computer production skills and hand-painted techniques that are not aimed at bag products.

The necessity of the heuristic task first. As mentioned above, we have made a clear explanation of the "heuristic" task, so in order to ensure the proposition that the idea of getting the right solution is less clear, we should weaken students on some issues, such as "how to make out a bag"; "what are the structures of a usual bag?"; "what are the features of the bag?"; "What functions does a bag need?", so as to prevent the subjects from getting a set of solutions for themselves by combining their own experience with the focus in the instructions, thereby leading to low levels of creativity in the final results.

Task contents. In a heuristic task, the task is executed as follows:

(1) The teacher prompts the task, and the concrete instructions are as follows:

I am the keynote teacher of this course. Before the beginning of the course, I would like to ask you to participate in an interactive game in order to experience the pleasure of being a designer. Please design a bag according to your preferences, and the form is unlimited. Please note that this task will not record your results. My focus is not on the outcome of the design, but for the freedom to experience this casual thinking. Please fill out a questionnaire about the design process at the end of the task.

(2) When the students make the design plan, the teachers also make their own plans together

(3) Teachers and students participate in an informal program discussion: All people (including teachers) are divided into two groups: one group is the suggestion group, and the other is the design presentation group. Each subject in the presentation group can communicate with any of the members of the suggestion group, while members of the suggestion group can only give positive comments or affirmation on the strengths of the works of the presentation group members. All exchanges are free to choose locations and exchange roles at the end. The recommended subjects need to be evaluated and documented.

(4) According to the suggestion of others and their new ideas, the subjects need to revise the plan again to form the new final plan, or they can choose not to modify the plan.

\section{Matters needing attention and theoretical basis in the task.}

(1) When the task is put forward, the teacher must make sure that the answer to the task is open and that attention should be given to avoid restrictive conditions, such as the steps and design procedure, etc.

(2) In the course of the task, it is necessary to ensure that there are no evaluation expectations for participants as described in the instructions above: "I'm not focusing on the final design, and I'm not going to score it."

(3) In the course of the task, teachers should also participate in the discussion as a design participant. There are two purposes for doing so: 1. Set an example. According to the theory of Creativity in Context, this will help drive the intrinsic motivation of the subjects. 2. Create a more "open" classroom atmosphere [7-8], encourage curiosity, exploration and self-directed learning, but not advocate scoring and authoritative instruction [2]. 
(4) This course is a specialized elective course, so students have the right to choose for the participation of the course. According to the conclusion of the experimental study of Amabile in 1982, the creativity score under this type of task would be higher if "no reward" was applied. As a result, teachers cannot refer to anything such as "reward" and "scoring criteria" when conducting a heuristic task [2]. If the course is a compulsory course, and then the situation is opposite (Figure 2).

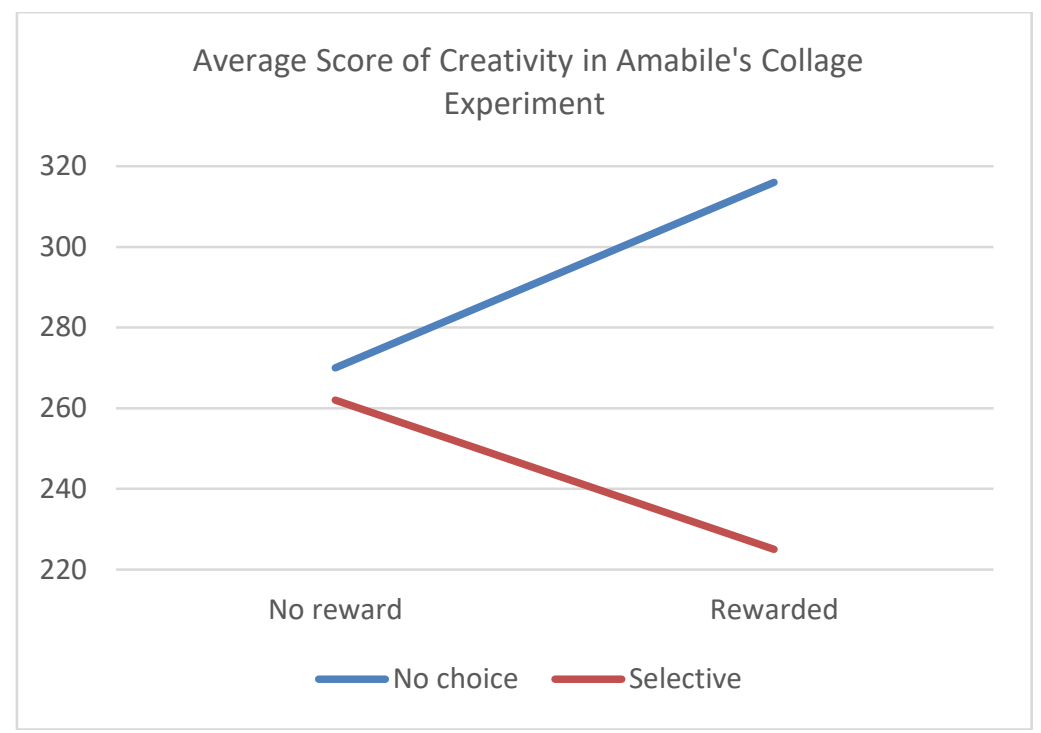

Figure 2. Average Score of Creativity in Collage Experiment[2]

(5) Students must be free to choose their creative expressions. The individual's intrinsic motivation will be elevated if he/she perceives that he/she has the right to choose some aspect of the task. [9] This conclusion is well proved in an experiment by Amabile and Judy Gitomer. [2]

(6) At the end of the task, when giving specific evaluation of each creative plan, the evaluation must be targeted and affirmative or positive comments on the individual's ability to do the task, and some unexpected rewards may also be presented. For example, the reward used in this study is that students with good performance can get internship opportunities in related enterprises. Unexpected rewards will allow participants to enhance their internal drive to the design of bags. Research shows that the individual's interest in an activity is positively related to the creativity shown. [2]

\subsection{Followed by the algorithmic task}

\subsubsection{Algorithmic task}

Amabile has repeatedly stressed in her book that theory of Creativity in Context applies only to heuristic tasks [2]. Algorithmic task processes are more appropriate to describe techniques in art creation. Although there is no explicit definition of algorithmic tasks in the text, some of the specific descriptions can also help us understand the characteristics of this task type. It is a "script" (Schank\&Abelson, 1977) or an algorithm that performs a task, that is, a fixed step that performs a task or solves a problem in a certain field [2]. If you can clearly and directly get the solution, and then the issue is an "algorithmic task". [2] It is certain that the "arithmetic" task means that when the task is executed, it can draw clear and direct steps to implement the task or solve the problem according to the previous knowledge and experience. 
The reasons for the baseline level and followed by the algorithmic task have been discussed in the above, so only the main contents of the algorithmic task will be explained here.

As shown in Figure 1, the product design process used in general teaching, namely, a series of teaching contents used to strengthen domain-relevant skills, [2] such as the sketch, functional structure analysis, man-machine engineering knowledge, computer modeling, drawing design and model production and graphic display production, are the algorithmic tasks described in this article, because the methods you need to learn these skills are step-by-step, and you have to connect them gradually with specific steps to achieve the desired learning requirements. "Market research" in the initial stage, the design, the implementation and subsequent statistical work of a questionnaire are tasks with a fixed format. Only some qualitative analysis (User Studies) processes are likely to be closer to what we call heuristic task processes.

\subsubsection{Algorithmic tasks in the bag design course}

In algorithmic tasks, we mainly adopt the task-oriented curriculum teaching model, which is commonly used in the traditional design education field, to bring the task of solving specific problems into the whole design process, and we can achieve the special training purpose of the students' performance techniques of bags, structure and manufacturing process of bags and computer modeling through the step-by-step implementation of the task.

This approach is designed to enable students to have certain domain-relevant skills in real time in the third stage of concrete implementation of outcomes, and allow students not reduce the design creativity performance because of the lack of technology in terms of the expression of the design plan.

Specific implementation steps are divided into: (1) Select an existing product on the market and present its problems (2) Put forward a variety of solutions according to the problem (3) Report the program and get the teacher's evaluation (4) Research on materials, man-machine engineering and other knowledge (5) Choose one of the options and optimize the final solution (6) The teacher evaluates the work. Different from heuristic tasks, at the beginning of the algorithmic task, the instructor will make a specific explanation and emphasis on the evaluation criteria, make a targeted positive evaluation in the middle and late phases of the task execution, or give some suggestions for improvement on the part of the work which is insufficient. According to Amabile's conclusion in the experiment of assessment-creativity in 1979, such evaluation expectations and technical focus will enable the subjects to perform well in technical scoring [2].

\section{Results}

\subsection{Results of heuristic task stage}

The results of the heuristic task stage can be summarized as follows:

(1) The subjects showed strong initiative and enthusiasm in the process of interactive review. According to the playback after class, the students generally reflected that it was interesting to work with teachers and comment on each other.

(2) Open propositional way enabled the students to put forward various ideas

(3) At the end of the heuristic task, many students showed a strong interest in becoming a bag designer because of the unexpected rewards, and several students expressed the idea of getting into my bag design studio as a volunteer.

\subsection{Results of algorithm task stage}

The results of the algorithm task stage can be summarized as follows:

(1) The subjects showed stable learning state when they have evaluation expectations, and they could learn and master the skills in related fields in accordance with the guidance procedure. 
(2) The mid-term evaluation recommendations were helpful to improve the skills of the subjects.

\subsection{Results of heuristic task outcomes in concrete implementation phase}

The results of the heuristic task outcomes in concrete implementation can be summarized as follows:

(1) After the final creative evaluation, the average score of the trial group was 83 points (the 560 point system of the CAT scale was rounded and translated into 100 points), which was slightly higher than the 76 points of the control group.

(2) One of the students in the trial group was below average, with a score of 64 , and the reason for this was still to be further studied.

\section{Conclusions}

Based on Amabile's theory of Creativity in Context, we made a tentative application in the bag product design course and conduct a trial with samples. Moreover, we proposed a segmented education model of "the heuristic task" first and followed by "the algorithmic task" to enhance product design and students' creativity level. A Consensual Technique for Creativity was applied to measure students' creativity. Results effectively proved that the segmented teaching method could promote the creativity of students in the bag design course. Thus, we summarized this method into a preliminary theoretical framework, as showed in figure 3.

The art design education can be divided into three main stages: (1) Heuristic task first (2) Followed by the algorithmic task (3) Implementation of heuristic task outcomes. The specific steps at each stage are shown in the green squares in the table, while the red word shows the corresponding theoretical basis at this stage.

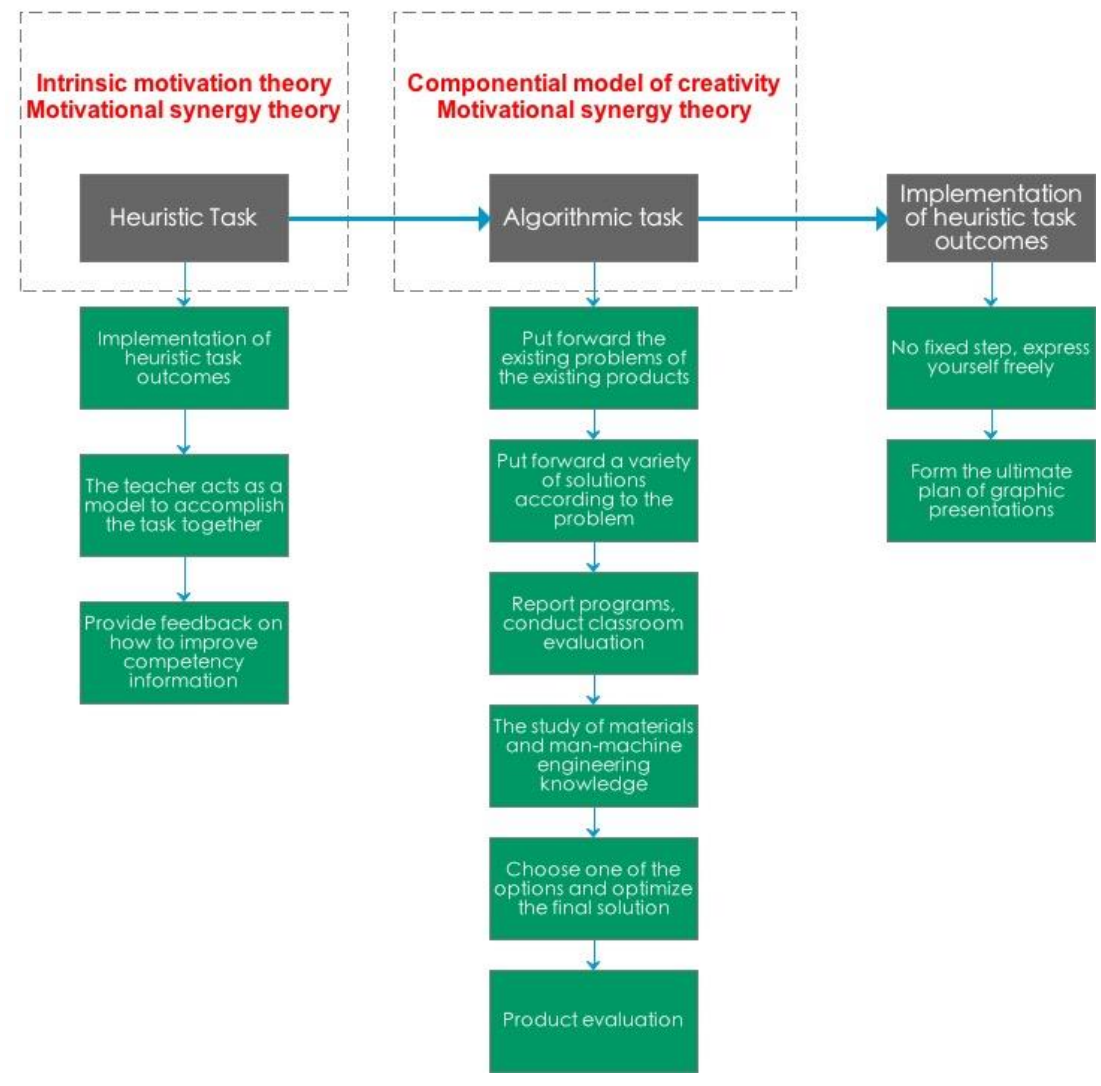


Figure 3. A segmented educational theory framework for effective creativity training in product design courses

\section{Acknowledgement}

Project Research Team of Intelligence Sustainable Package Design Support by Shanghai Summit Discipline in Design; Foundation item: Chenguang Plan (13CG74) sponsor

\section{References}

1. T. E. Chang, Psychology of Creativity: Theories and Methods of Creation, (Hebei People's Publishing House, Hebei, 2000)

2. T. M. Amabile, Creativity in context: Update to the Social Psychology of Creativity (Westview, Boulder, CO, 1996)

3. H. Y. Sun, Creativity Psychology (Beijing Normal University Publishing Group, Beijing, 2016)

4. Y. L. Gui, C. Yang, Product Design (China Light Industry Press, Beijing, 2016)

5. T. M. Amabile, Creativity in context: Update to the Social Psychology of Creativity (Sichuan People's Publishing House, Sichuan, 2017)

6. A. Osborn, Applied imagination: Principles and Procedures of creative thinking (Scribner's, New York,1963)

7. C. T. Ramey, V. Piper, Child Development, 45, 557-560 (1974)

8. M. Hickey, JRME, 49, 234-244 (2001)

9. M. Zuckerman, J. Porac, D. Lathin, R. Smith, E. L. Deci, Personality and Social Psychology Bulletin, 4, 443-446 (1978) 